\title{
Transverse complex magnetic susceptibility of single-domain ferromagnetic particles with uniaxial anisotropy subjected to a longitudinal uniform magnetic field
}

\author{
Yu P. Kalmykov \\ Institute of Radio Engineering and Electronics of the Russian Academy of Sciences, Vvedenskii Square 1, \\ Fryazino, Moscow Region, 141120, Russian Federation \\ W. T. Coffey* \\ Department of Electronic and Electrical Engineering, Trinity College, Dublin 2, Ireland
}

(Received 20 December 1996)

\begin{abstract}
The infinite hierarchy of differential-recurrence relations for the equilibrium transverse correlation functions appropriate to magnetic relaxation of single-domain ferromagnetic particles with uniaxial anisotropy subjected to a uniform external magnetic field $H_{0}$ is derived by averaging Gilbert's equation. Exact expressions in terms of matrix continued fractions for the transverse complex magnetic susceptibility are obtained with the aid of linear-response theory by solving the infinite hierarchy. The principal features of the spectra are emphasized in figures showing the real and imaginary parts of the complex magnetic susceptibility. The accuracy and the range of the applicability of analytical results based on the effective eigenvalue method is established. It is shown that this method provides in general a good approximation to the exact solution with the exception of the range of low-to-intermediate barrier heights of the anisotropy potential where at small $H_{0}$ there exists essentially a spread of the precession frequencies of the magnetization. [S0163-1829(97)03229-3]
\end{abstract}

\section{INTRODUCTION}

A single-domain ferromagnetic particle with a uniaxial anisotropy is characterized by an internal potential which has two stable stationary points with a potential barrier between them. If the particle is sufficiently fine (and hence the potential barrier is low), the vector of magnetization $\mathbf{M}$ may undergo a rotation due to thermal agitation, surmounting the barrier, as described by Néel. ${ }^{1}$ The thermal instability of the magnetization arises from the so-called superparamagnetism ${ }^{2}$ because each fine particle behaves as an enormous paramagnet of magnetic moment $10^{4}-10^{5} \mu_{\mathrm{B}}$. The thermal fluctuations and relaxation of the magnetization of a single-domain particle currently merits attention in view of its importance in the context of magnetic recording media ${ }^{3}$ and paleomagnetism. $^{4}$

The behavior of the magnetization of a single-domain ferromagnetic particle has been the subject of much study. Landau and Lifshitz in their 1935 paper $^{5}$ gave an equation of motion describing the average behavior of the magnetization. Gilbert in his 1955 paper $^{6}$ presented a similar equation of motion. Brown ${ }^{7}$ extended these equations of motion to describe not the average but the dynamic behavior of the magnetization of an individual single-domain ferromagnetic particle. He based his work on the Langevin equation approach to the theory of Brownian motion. Brown took as the Langevin equation, Gilbert's equation transformed to LandauLifshitz form. He was able to construct from it the underlying probability density diffusion equation, which is the Fokker-Planck equation. In order to accomplish this, Brown wrote down the equation of motion in spherical polar coordinates. He then used the methods of Wang and Uhlenbeck ${ }^{8}$ combined with the Stratonovich ${ }^{9}$ definition of the derivative of a stochastic variable to construct the Fokker-Planck equation for the distribution function $W(\{\mathbf{M}\}, t)$ of the orienta- tions of the magnetization vector $\mathbf{M}$ in these coordinates. The Fokker-Planck equation in the case of axial symmetry can be solved by the method of separation of the variables. The separation procedure gives rise to an equation of the Sturm-Liouville type. An alternative approach to the problem which is not confined to axial symmetry is to expand $W$ as a series of spherical harmonics so yielding an infinite hierarchy of linear differential-recurrence equations for averaged spherical harmonics. The infinite hierarchy can be then solved by finding eigenvalues and eigenvectors of the system matrix or, much more efficiently, by a matrix continuedfraction method. ${ }^{10}$ This hierarchy can also be obtained by directly averaging Gilbert's equation without recourse to the Fokker-Planck equation. ${ }^{11,12}$

A basic model in the study of the superparamagnetism is when an external uniform magnetic field $H_{0}$ of arbitrary strength is superimposed on the anisotropy potential field. $^{1,13,14}$ In the simplest case of the uniaxial anisotropy the free-energy density $V$ of a single-domain particle is given by

$$
V=-K \cos ^{2} \vartheta-\left(\mathbf{M} \cdot \mathbf{H}_{0}\right)
$$

where $K$ is the anisotropy constant and $\vartheta$ is the polar angle. In general such a field can only be applied at some angle to the easy axis of magnetization since the axis is in a random position. However, in order to preserve the axial symmetry of the problem and its attendant mathematical simplifications it is often assumed that $H_{0}$ is applied along the polar axis. Initiated by $\mathrm{Neel}^{1}$ and Stoner and Wohlfahrt ${ }^{14}$ this problem has been recently studied in Refs. 15-19. However, these studies were mainly confined to the longitudinal relaxation so that only in Refs. 15 and 19 were approximate transverse relaxation solutions for several particular cases obtained by dint of the effective eigenvalue (moment) method (for recent reviews of the method, see Refs. 20 and 21, and references 
cited therein). This method was applied by Raŭkher and Shliomis ${ }^{22}$ to the study of relaxation and ferromagnetic resonance in the uniaxial anisotropy potential at $H_{0}=0$, and was recently extended ${ }^{20,23}$ to more complicated anisotropy potentials such as a cubic anisotropy. However, the formal application of this approximate method to the transverse relaxation may not be deemed completely satisfactory as the results obtained for the transverse response were not compared with the exact solutions, so that the accuracy of these results remains unknown. Indeed, we shall demonstrate in the present paper that the effective eigenvalue method fails to describe the transverse relaxation at low to moderate barrier heights and $H_{0}=0$.

The primary goal of this paper is to evaluate the transverse complex magnetic susceptibility $\chi_{\perp}(\omega)$ of a system of noninteracting single-domain ferromagnetic particles subjected to a constant magnetic field. We obtain (with the aid of linear-response theory) the exact solutions for $\chi_{\perp}(\omega)$ in terms of matrix continued fractions. In order to obtain these results we shall use the approach of Coffey, Kalmykov, and Waldron $^{12}$ for the solution of the infinite hierarchy of differential-recurrence relations which has already allowed us to obtain the exact solution for the longitudinal relaxation. ${ }^{16}$ This approach is based on matrix continued fractions and essentially constitutes a further development of Risken's method. ${ }^{10}$ It has also been used in the theory of dielectric and Kerr effect relaxation. ${ }^{24,25}$

Before proceeding we must first summarize the principal results of linear-response theory (Ref. 10, Chap. 7). The application of this theory to axially symmetric magnetic problems predicts that the decay of the magnetization $\langle\mathbf{M}\rangle(t)$ of a system of noninteracting single-domain ferromagnetic particles, when a small constant external field $H_{1}$ $\left[v\left(\mathbf{M} \cdot \mathbf{H}_{1}\right) / k T \ll 1\right]$ has been switched off at time $t=0$, is

$$
\left\langle M_{\|}\right\rangle(t)-\left\langle M_{z}\right\rangle_{0}=\chi_{\|} H_{1} C_{\|}(t),
$$

(for the case of the longitudinal relaxation, $H_{0} \| H_{1}$ ) and

$$
\left\langle M_{\perp}\right\rangle(t)=\chi_{\perp} H_{1} C_{\perp}(t),
$$

(for the case of the transverse relaxation, $H_{0} \perp H_{1}$ ), where

$$
\chi_{\|}=\frac{\nu^{2} N_{0}}{k T}\left[\left\langle M_{z}^{2}\right\rangle_{0}-\left\langle M_{z}\right\rangle_{0}^{2}\right]
$$

and

$$
\chi_{\perp}=\frac{\nu^{2} N_{0}}{k T}\left\langle M_{x}^{2}\right\rangle_{0}=\frac{\nu^{2} N_{0}}{k T}\left\langle M_{y}^{2}\right\rangle_{0}
$$

are the components of the static magnetic susceptibility tensor, $N_{0}$ is the number of particles per unit volume, $\nu$ is the volume of the particle. Also,

$$
C_{\|}(t)=\frac{\left\langle M_{z}(0) M_{z}(t)\right\rangle_{0}-\left\langle M_{z}\right\rangle_{0}^{2}}{\left\langle M_{z}^{2}\right\rangle_{0}-\left\langle M_{z}\right\rangle_{0}^{2}}
$$

and

$$
C_{\perp}(t)=\frac{\left\langle M_{x}(0) M_{x}(t)\right\rangle_{0}}{\left\langle M_{x}^{2}\right\rangle_{0}}=\frac{\left\langle M_{y}(0) M_{y}(t)\right\rangle_{0}}{\left\langle M_{y}^{2}\right\rangle_{0}}
$$

are the normalized autocorrelation functions of the longitudinal and transverse components of the magnetization of the particle, respectively, $M_{x}, M_{y}, M_{z}$ are the Cartesian components of $\mathbf{M}$, and the brackets \langle\rangle$_{0}$ designate the equilibrium ensemble average. According to linear-response theory ${ }^{10}$ the corresponding components of the complex magnetic susceptibility tensor $\chi_{\|}(\omega)$ and $\chi_{\perp}(\omega)$ are given by

$$
\begin{gathered}
\chi_{\gamma}(\omega)=\chi_{\gamma}^{\prime}(\omega)-i \chi_{\gamma}^{\prime \prime}(\omega)=\chi_{\gamma}\left[1-i \omega \int_{0}^{\infty} e^{-i \omega t} C_{\gamma}(t) d t\right] \\
(\gamma=\|, \perp) .
\end{gathered}
$$

The longitudinal complex susceptibility when a uniform magnetic field $H_{0}$ of arbitrary strength is superimposed on the uniaxial anisotropy potential field has been evaluated in Ref. 16. In order to evaluate the transverse susceptibility we must first calculate the equilibrium correlation function $C_{\perp}(t)$ which is more conveniently accomplished directly from Gilbert's equation rather than from the Fokker-Planck one. ${ }^{11}$ Thus, we bypass the Fokker-Planck equation entirely.

\section{DERIVATION OF DIFFERENTIAL-RECURRENCE RELATIONS FROM GILBERT'S EQUATION AUGMENTED BY A RANDOM-FIELD TERM}

Gilbert's equation in the presence of thermal agitation is ${ }^{6}$

$$
\frac{d}{d t} \mathbf{M}(t)=\gamma[\mathbf{M}(t) \times[\mathbf{H}(t)+\mathbf{h}(t)-\eta \dot{\mathbf{M}}(t)]],
$$

where $\gamma$ is the gyromagnetic ratio, $\eta$ is the damping parameter, $\mathbf{H}(t)$ is the magnetic field acting on the particle which may consist of externally applied magnetic fields, the crystalline anisotropy field, and a random Gaussian white-noise field $\mathbf{h}(t)$, which has the properties

$$
\overline{h_{i}(t)}=0,
$$

$$
\overline{h_{i}(t) h_{j}\left(t^{\prime}\right)}=(2 k T \eta / \nu) \delta_{i j} \delta\left(t-t^{\prime}\right) .
$$

Here the overbar means the statistical average over an ensemble of particles which all have at time $t$ the same magnetization M. If $V(\{\mathbf{M}\})$ is the free energy per unit volume expressed as a function of components of $\mathbf{M}$, then

$$
\mathbf{H}=-\frac{\partial}{\partial \mathbf{M}} V(\{\mathbf{M}\}) .
$$

It is assumed throughout this analysis that the magnetization is uniform inside the particle and only the orientation and not the magnitude of the magnetization is subject to variations. The assumptions made in the derivation of Gilbert's equation were discussed elsewhere (e.g., Ref. 26).

Gilbert's equation (2.1) may be rearranged explicitly (as shown, e.g., in Refs. 2 and 19) using the properties of the triple product formula to yield that equation in the Landau-Lifshitz ${ }^{5}$ form:

$$
\begin{aligned}
\frac{d}{d t} \mathbf{M}(t)= & M_{s} g^{\prime}[\mathbf{M}(t) \times[\mathbf{H}(t)+\mathbf{h}(t)]] \\
& +h^{\prime}[[\mathbf{M}(t) \times[\mathbf{H}(t)+\mathbf{h}(t)]] \times \mathbf{M}(t)],
\end{aligned}
$$


where

$$
\alpha=\gamma \eta M_{s}
$$

$$
g^{\prime}=\frac{\gamma}{\left(1+\alpha^{2}\right) M_{s}}, \quad h^{\prime}=\frac{\gamma \alpha}{\left(1+\alpha^{2}\right) M_{s}}=\alpha g^{\prime},
$$

$M_{s}$ is the saturation magnetization, and the damping constant $\alpha$ is given by
Equation (2.4) is of the same mathematical form as the Landau-Lifshitz equation ${ }^{5}$ except that both parameters $g^{\prime}$ and $h^{\prime}$ depend on $\alpha$.

Expanding Eq. (2.4) in its Cartesian components we have

$$
\begin{aligned}
\frac{1}{h^{\prime} M_{s}} \frac{d}{d t} u_{x}(t)= & {\left[1-u_{x}^{2}(t)\right] h_{x}(t)-\left[a^{-1} u_{z}(t)+u_{x}(t) u_{y}(t)\right] h_{y}(t)+\left[\alpha^{-1} u_{y}(t)-u_{z}(t) u_{x}(t)\right] h_{z}(t)+\left[1-u_{x}^{2}(t)\right] H_{x}(t) } \\
& -\left[\alpha^{-1} u_{z}(t)+u_{x}(t) u_{y}(t)\right] H_{y}(t)+\left[\alpha^{-1} u_{y}(t)-u_{z}(t) u_{x}(t)\right] H_{z}(t), \\
\frac{1}{h^{\prime} M_{s}} \frac{d}{d t} u_{y}(t)= & {\left[\alpha^{-1} u_{z}(t)-u_{x}(t) u_{y}(t)\right] h_{x}(t)+\left[1-u_{y}^{2}(t)\right] h_{y}(t)-\left[\alpha^{-1} u_{x}(t)+u_{y}(t) u_{x}(t)\right] h_{z}(t)+\left[\alpha^{-1} u_{z}(t)\right.} \\
& \left.-u_{x}(t) u_{y}(t)\right] H_{x}(t)+\left[1-u_{y}^{2}(t)\right] H_{y}(t)-\left[\alpha^{-1} u_{x}(t)+u_{y}(t) u_{x}(t)\right] H_{z}(t), \\
\frac{1}{h^{\prime} M_{s}} \frac{d}{d t} u_{z}(t)= & -\left[\alpha^{-1} u_{y}(t)+u_{x}(t) u_{z}(t)\right] h_{x}(t)+\left[\alpha^{-1} u_{x}(t)-u_{y}(t) u_{z}(t)\right] h_{y}(t)+\left[1-u_{z}^{2}(t)\right] h_{z}(t)-\left[\alpha^{-1} u_{y}(t)\right. \\
& \left.+u_{x}(t) u_{z}(t)\right] H_{x}(t)+\left[\alpha^{-1} u_{x}(t)-u_{y}(t) u_{z}(t)\right] H_{y}(t)+\left[1-u_{z}^{2}(t)\right] H_{z}(t),
\end{aligned}
$$

where

$$
\begin{gathered}
u_{x}=M_{x} / M_{s}=\sin \vartheta \cos \varphi, \quad u_{y}=M_{y} / M_{s}=\sin \vartheta \sin \varphi, \\
u_{z}=M_{z} / M_{s}=\cos \vartheta .
\end{gathered}
$$

The set of stochastic differential equations (2.7)-(2.9) contains multiplicative noise terms $h_{i}(t) u_{i}(t) u_{k}(t)$. This poses an interpretation problem for these equations as has been discussed in Refs. 10 and 12. We recall, taking the set of the Langevin equations for the $N$ stochastic variables $\{\xi(t)\}=\left\{\xi_{1}(t), \xi_{2}(t), \ldots, \xi_{N}(t)\right\}$ :

$\frac{d \xi_{i}(t)}{d t}=h_{i}(\{\xi(t)\}, t)+g_{i j}(\{\xi(t)\}, t) \Gamma_{j}(t), \quad(i, j=1, \ldots, N)$,

with

$$
\begin{gathered}
\overline{\Gamma_{i}(t)}=0, \\
\overline{\Gamma_{i}(t) \Gamma_{j}\left(t^{\prime}\right)}=2 D \delta_{i j} \delta\left(t-t^{\prime}\right),
\end{gathered}
$$

and interpreting them as Stratonovich equations, that the averaged equations for the sharp values $\xi_{i}(t)=x_{i}$ at time $t$ $\operatorname{are}^{10,12}$

$$
\begin{aligned}
\frac{d x_{i}}{d t}=\lim _{\tau \rightarrow 0} \frac{\overline{\xi_{i}(t+\tau)-x_{i}}}{\tau} \\
=h_{i}(\{\mathbf{x}\}, t)+D g_{k j}(\{\mathbf{x}\}, t) \frac{\partial}{\partial x_{k}} g_{i j}(\{\mathbf{x}\}, t), \\
(i, j=1, \ldots, N),
\end{aligned}
$$

where $\xi_{i}(t+\tau)(\tau>0)$ is the solution of Eq. (2.10) with the initial conditions $\xi_{i}(t)=x_{i}$. In Eqs. (2.10) and (2.12) the summation over $j$ and $k$ is understood (Einstein's notation). The proof of Eq. (2.12) can be found elsewhere (see Ref. 10, pp. 54 and 55). We remark that just as for dielectric relaxation $^{24}$ we shall always use the Stratonovich definition $^{9,27}$ of the average of the multiplicative noise term here as that definition always constitutes the mathematical idealization of the physical relaxation process. Thus, it is unnecessary to transform the Langevin equations (2.7)-(2.9) to Itô equations (e.g., Ref. 27). Moreover, we can apply the methods of ordinary analysis. ${ }^{27}$

In like manner we can prove that the averaged equation for an arbitrary differentiable function $f(\{\xi\})$ has the following form (see Appendix A):

$$
\begin{aligned}
\frac{d f(\{\mathbf{x}\})}{d t}= & \lim _{\tau \rightarrow 0} \frac{\overline{f(\{\xi(t+\tau)\})-f(\{\mathbf{x}\})}}{\tau} \\
= & h_{i}(\{\mathbf{x}\}, t) \frac{\partial}{\partial x_{i}} f(\{\mathbf{x}\})+D g_{k j}(\{\mathbf{x}\}, t) \\
& \times \frac{\partial}{\partial x_{k}}\left[g_{i j}(\{\mathbf{x}\}, t) \frac{\partial}{\partial x_{i}} f(\{\mathbf{x}\})\right],
\end{aligned}
$$

where summation over $i, j$, and $k$ is also understood.

In the study of the orientation relaxation the quantities of interest are the spherical harmonics $X_{n m}$ defined as

$$
X_{n m}=e^{i m \varphi} P_{n}^{m}(\cos \vartheta)=e^{i m \varphi}\left(1-\cos ^{2} \vartheta\right)^{m / 2} \frac{d^{m} P_{n}(\cos \vartheta)}{d \cos \vartheta^{m}},
$$


where $P_{n}(x)$ and $P_{n}^{m}(x)$ are the Legendre polynomials and the associated Legendre functions of the first kind, respectively. ${ }^{28}$ The $X_{n m}$ are expressed in terms of $u_{x}, u_{y}, u_{z}$ as follows:

$$
X_{n m}=\left(u_{x}+i u_{y}\right)^{m} \frac{d^{m} P_{n}\left(u_{z}\right)}{d u_{z}^{m}} .
$$

Noting that according to the Stratonovich definition the conventional rules of transformation of a stochastic variable (ordinary calculus) can be used ${ }^{27}$ and taking into account the theorem Eq. (2.13) with

$$
\begin{aligned}
\frac{d}{d t} X_{n m}= & m\left(u_{x}+i u_{y}\right)^{m-1} \frac{d^{m} P_{n}\left(u_{z}\right)}{d u_{z}^{m}} \frac{d}{d t} u_{x} \\
& +i m\left(u_{x}+i u_{y}\right)^{m-1} \frac{d^{m} P_{n}\left(u_{z}\right)}{d u_{z}^{m}} u_{y} \\
& +\left(u_{x}+i u_{y}\right)^{m} \frac{d^{m+1} P_{n}\left(u_{z}\right)}{d u_{z}^{m+1}} \frac{d}{d t} u_{z},
\end{aligned}
$$

we can obtain from Eqs. (2.7), (2.8), and (2.9) the equation of motion of the sharp values of spherical harmonics $X_{n m}$ (Refs. 11 and 12):

$$
\begin{aligned}
2 \tau_{N} \frac{d}{d t} X_{n m}= & \frac{\nu M_{s}}{k T}\left\{\left(u_{x}+i u_{y}\right)^{m} \frac{d^{m+1} P_{n}\left(u_{z}\right)}{d u_{z}^{m+1}}\left[H_{z}-u_{z}(\mathbf{u} \cdot \mathbf{H})+\alpha^{-1}\left(u_{x} H_{y}-u_{y} H_{x}\right)\right]+m\left(u_{x}+i u_{y}\right)^{m-1} \frac{d^{m} P_{n}\left(u_{z}\right)}{d u_{z}^{m}}\right. \\
& \left.\times\left[\left(H_{x}+i H_{y}\right)\left(1+i \alpha^{-1} u_{z}\right)-\left(u_{x}+i u_{y}\right)\left((\mathbf{u} \cdot \mathbf{H})+i \alpha^{-1} H_{z}\right)\right]\right\}+\eta h^{\prime} M_{s}^{2} g_{k j} \frac{\partial}{\partial u_{k}}\left[m\left(u_{x}+i u_{y}\right)^{m-1} \frac{d^{m} P_{n}\left(u_{z}\right)}{d u_{z}^{m}}\right. \\
& \left.\times\left(g_{x j}+i g_{y j}\right)+\left(u_{x}+i u_{y}\right)^{m} \frac{d^{m+1} P_{n}\left(u_{z}\right)}{d u_{z}^{m+1}} g_{z j}\right]
\end{aligned}
$$

where

$$
\begin{aligned}
& g_{x x}=1-u_{x}^{2}, \quad g_{x y}=-\alpha^{-1} u_{z}-u_{y} u_{x}, \quad g_{x z}=\alpha^{-1} u_{y}-u_{z} u_{x}, \\
& g_{y x}=\alpha^{-1} u_{z}-u_{y} u_{x}, \quad g_{y y}=1-u_{y}^{2}, \quad g_{y z}=-\alpha^{-1} u_{x}-u_{y} u_{z}, \\
& g_{z x}=-\alpha^{-1} u_{y}-u_{z} u_{x}, \quad g_{z y}=\alpha^{-1} u_{x}-u_{y} u_{z}, \quad g_{z z}=1-u_{z}^{2},
\end{aligned}
$$

and the relaxation time $\tau_{N}$ is given by

$$
\tau_{N}=\frac{\nu}{2 k T h^{\prime}}
$$

We remark that all the $X_{n m}$ and $H_{i}$ in Eq. (2.16) are in general functions of $u_{x}, u_{y}, u_{z}$. Also $u_{x}, u_{y}, u_{z}$ in Eq. (2.16) and $u_{x}(t), u_{y}(t), u_{z}(t)$ in Eqs. (2.7)-(2.9) have different meanings, namely, $u_{x}(t), u_{y}(t), u_{z}(t)$ in Eqs. (2.7)-(2.9) are stochastic variables, while $u_{x}, u_{y}, u_{z}$ in Eq. (2.16) are the sharp (definite) values $u_{k}(t)=u_{k}$ at time $t$. Instead of using different symbols for the two quantities we have distinguished sharp values at time $t$ from stochastic variables by deleting the time argument as in Ref. 10.

The right-hand side of Eq. (2.16) consists of two terms, namely, the deterministic drift and the noise-induced (or spurious) drift. These terms can be considerably simplified after some algebra. ${ }^{11}$ In particular, the spurious drift is given by

$$
\eta h^{\prime} M_{s}^{2} g_{k j} \frac{\partial}{\partial u_{k}}\left[m\left(u_{x}+i u_{y}\right)^{m-1} \frac{d^{m} P_{n}\left(u_{z}\right)}{d u_{z}^{m}}\left(g_{x y}+i g_{y j}\right)+\left(u_{x}+i u_{y}\right)^{m} \frac{d^{m+1} P_{n}\left(u_{z}\right)}{d u_{z}^{m+1}} g_{z j}\right]=-n(n+1) X_{n m} .
$$

Thus, we obtain

$$
\begin{aligned}
2 \tau_{N} \frac{d}{d t} X_{n m}+n(n+1) X_{n m}= & \frac{\nu M_{s}\left(H_{x}+i H_{y}\right)}{2 k T(2 n+1)}\left[n(n-m+1)(n-m+2) X_{n+1 m-1}+i \alpha^{-1}(2 n+1)(n-m+1)(n+m) X_{n m-1}\right. \\
& \left.+(n+1)(n+m-1)(n+m) X_{n-1 m-1}\right]-\frac{\nu M_{s}\left(H_{x}-i H_{y}\right)}{2 k T(2 n+1)}\left[n X_{n+1 m+1}-i \alpha^{-1}(2 n+1) X_{n m+1}\right. \\
& \left.+(n+1) X_{n-1 m+1}\right]-\frac{\nu M_{s} H_{z}}{k T(2 n+1)}\left[n(n-m+1) X_{n+1 m}+i \alpha^{-1} m(2 n+1) X_{n m}\right. \\
& \left.-(n+1)(n+m) X_{n-1 m}\right] .
\end{aligned}
$$

Equation (2.20) is valid for any anisotropy potential.

Equation (2.20) can be further simplified for a uniaxial magnetic anisotropy field with uniaxial anisotropy energy density 


$$
V=-K u_{z}^{2}=-K \cos ^{2} \vartheta
$$

where $\vartheta$ is the angle between $\mathbf{M}$ and the positive $z$ axis, superimposed on a strong constant magnetic field $H_{0}$ applied along the $z$ direction. Thus the field $\mathbf{H}$ has only a $z$ component given by

$$
\mathbf{H}=\left(H_{0}+\frac{2 K}{M_{s}} u_{z}\right) \mathbf{k} .
$$

On substituting Eq. (3.24) into Eq. (3.22) and on using the equalities for the associated Legendre functions ${ }^{29}$

$$
\begin{gathered}
\sqrt{1-x^{2}} P_{n}^{m+1}(x)-(n+m+1) x P_{n}^{m}(x)+(n-m+1) P_{n+1}^{m}(x)=0, \\
(2 n+1) x P_{n}^{m}(x)=(n-m+1) P_{n+1}^{m}(x)+(n+m) P_{n-1}^{m}(x),
\end{gathered}
$$

we have

$$
\begin{aligned}
\tau_{N} \frac{d}{d t} X_{n m}+ & {\left[\frac{n(n+1)}{2}+\frac{i \xi m}{2 \alpha}-\sigma \frac{n(n+1)-3 m^{2}}{(2 n-1)(2 n+3)}\right] X_{n m} } \\
= & \xi\left[\frac{(n+m)}{2 n+1}\left(\frac{n+1}{2}-\frac{i m \sigma}{\alpha \xi}\right) X_{n-1 m}-\frac{(n-m+1)}{2 n+1}\left(\frac{n}{2}+\frac{i m \sigma}{\alpha \xi}\right) X_{n+1 m}\right]+\sigma\left[\frac{(n+1)(n+m)(n+m-1)}{(2 n-1)(2 n+1)} X_{n-2 m}\right. \\
& \left.-\frac{n(n-m+1)(n-m+2)}{(2 n+1)(2 n+3)} X_{n+2 m}\right]
\end{aligned}
$$

where

$$
\sigma=\frac{\nu K}{k T}, \quad \xi=\frac{\nu M_{s} H_{0}}{k T} .
$$

The quantities $X_{n m}$ in Eq. (2.25) are functions of the sharp values $u_{k}$ which are themselves random variables with the distribution (probability density) function $W$. Therefore we must also average Eq. (2.25) over $W .^{12}$ In the absence of external perturbations, the system is at equilibrium with Boltzmann distribution function $W_{0}$ given by

$$
W_{0}(\vartheta)=\frac{1}{Z} \exp \left(-\frac{\nu V}{k T}\right)=\frac{1}{Z} \exp \left(\xi \cos \vartheta+\sigma \cos ^{2} \vartheta\right)
$$

where $Z$ is the normalizing constant (the partition function). As the equilibrium distribution (2.27) is independent of the azimuthal angle $\varphi$, all equilibrium ensemble averages $\left\langle X_{n m}\right\rangle_{0}$ vanish for $m \neq 0$, and \langle\rangle$_{0}$ designates the equilibrium averaging defined as

$$
\langle A\rangle_{0}=\frac{1}{Z} \int_{0}^{2 \pi} \int_{0}^{\pi} A(\vartheta, \varphi) e^{-\nu V(\vartheta) / k T} \sin \vartheta d \vartheta d \varphi
$$

We may construct from Eq. (2.25) an infinite hierarchy of differential-recurrence equations for any equilibrium correlation functions which may be desired. In particular, on multiplying Eq. (2.25) by $\operatorname{Re}\left\{X_{1 m}(0)\right\}$, and averaging the resulting equation over the equilibrium distribution function $W_{0}$ at the instant $t=0$, we obtain the hierarchy for the equilibrium transverse correlation functions:

$$
\begin{aligned}
& \tau_{N} \frac{d}{d t} f_{n, m}(t)+\left[\frac{n(n+1)}{2}+\frac{i \xi m}{2 \alpha}-\sigma \frac{n(n+1)-3 m^{2}}{(2 n-1)(2 n+3)}\right] f_{n, m}(t) \\
&=\xi\left[\frac{(n+m)}{2 n+1}\left(\frac{n+1}{2}-\frac{i m \sigma}{\alpha \xi}\right) f_{n-1, m}(t)-\frac{(n-m+1)}{2 n+1}\left(\frac{n}{2}+\frac{i m \sigma}{\alpha \xi}\right) f_{n+1, m}(t)\right]+\sigma\left[\frac{(n+1)(n+m)(n+m-1)}{(2 n-1)(2 n+1)} f_{n-2, m}(t)\right. \\
&\left.-\frac{n(n-m+1)(n-m+2)}{(2 n+1)(2 n+3)} f_{n+2, m}(t)\right]
\end{aligned}
$$

where $f_{n, m}(t)$ is the transverse correlation function defined as

$$
f_{n, m}(t)=\left\langle\operatorname{Re}\left\{X_{1 m}(0)\right\} X_{n m}(t)\right\rangle_{0}
$$


Equation (2.28) is valid for any $m$. However, for the problem under consideration it is enough to consider this equation for $m=1$ only because the transverse autocorrelation function $C_{\perp}(t)$ and complex magnetic susceptibility $\chi_{\perp}(\omega)$ are expressed in terms of $f_{1,1}(t)$ and its one-sided Fourier transform $\widetilde{f}_{1,1}(i \omega)$, viz.

$$
\begin{gathered}
C_{\perp}(t)=\frac{1}{f_{1,1}(0)} \operatorname{Re}\left[f_{1,1}(t)\right], \\
\chi_{\perp}(\omega)=\chi_{\perp}^{\prime}(\omega)-i \chi_{\perp}^{\prime \prime}(\omega)=\chi_{\perp}\left\{1-\frac{i \omega}{2 f_{1,1}(0)}\left[\widetilde{f}_{1,1}(i \omega)+\widetilde{f}_{1,1}^{*}(-i \omega)\right]\right\},
\end{gathered}
$$

where the asterisk denotes the complex conjugate,

$$
\chi_{\perp}=\frac{\nu^{2} M_{s}^{2} N_{0}}{3 k T}\left(1-\left\langle P_{2}\right\rangle_{0}\right)
$$

and

$$
\widetilde{f}_{n, m}(i \omega)=\int_{0}^{\infty} f_{n, m}(t) e^{-i \omega t} d t
$$

\section{EVALUATION OF THE COMPLEX TRANSVERSE SUSCEPTIBILITY IN TERMS OF MATRIX CONTINUED FRACTIONS}

For $m=1$ Eq. (2.28) can be transformed into the matrix three-term differential-recurrence equation

$$
\tau_{N} \frac{d}{d t} \mathbf{C}_{n}(t)=\mathbf{Q}_{n}^{-} \mathbf{C}_{n-1}(t)+\mathbf{Q}_{n} \mathbf{C}_{n}(t)+\mathbf{Q}_{n}^{+} \mathbf{C}_{n+1}(t)
$$

where

$$
\mathbf{C}_{n}(t)=\left(\begin{array}{c}
f_{2 n-1,1}(t) \\
f_{2 n, 1}(t)
\end{array}\right)=\left(\begin{array}{c}
\left\langle\sin \vartheta(0) \cos \varphi(0) X_{2 n-1,1}(t)\right\rangle_{0} \\
\left\langle\sin \vartheta(0) \cos \varphi(0) X_{2 n, 1}(t)\right\rangle_{0}
\end{array}\right)
$$

and

$$
\begin{aligned}
& \mathbf{Q}_{n}^{-}=\left(\begin{array}{cc}
\sigma \frac{4 n^{2}(2 n-1)}{(4 n-1)(4 n-3)} & \frac{2 n}{(4 n-1)}\left[n \xi-\frac{i \sigma}{\alpha}\right] \\
0 & \sigma \frac{2 n(2 n+1)^{2}}{(4 n-1)(4 n+1)}
\end{array}\right), \\
& \mathbf{Q}_{n}=\left(\begin{array}{cc}
\sigma \frac{2 n(2 n-1)-3}{(4 n-3)(4 n+1)}-n(2 n-1)-\frac{i \xi}{2 \alpha} & -\frac{(2 n-1)}{(4 n-1)}\left[\xi\left(n-\frac{1}{2}\right)+\frac{i \sigma}{\alpha}\right] \\
\frac{(2 n+1)}{(4 n+1)}\left[\xi\left(n+\frac{1}{2}\right)-\frac{i \sigma}{\alpha}\right] & \sigma \frac{2 n(2 n+1)-3}{(4 n-1)(4 n+3)}-n(2 n+1)-\frac{i \xi}{2 \alpha}
\end{array}\right), \\
& \mathbf{Q}_{n}^{+}=\left(\begin{array}{cc}
-\sigma \frac{2 n(2 n-1)^{2}}{(4 n-1)(4 n+1)} & 0 \\
-\frac{2 n}{(4 n+1)}\left[n \xi+\frac{i \sigma}{\alpha}\right] & -\sigma \frac{4 n^{2}(2 n+1)}{(4 n+1)(4 n+3)}
\end{array}\right)
\end{aligned}
$$

On applying the general method of solution of matrix three term differential-recurrence Eq. (3.1), suggested in Refs. 12 and 30, we obtain the exact solution for the onesided Fourier transform $\widetilde{\mathbf{C}}_{1}(i \omega)$ in terms of matrix continued fractions

$$
\left(\begin{array}{c}
\tilde{f}_{1,1}(i \omega) \\
\widetilde{f}_{2,1}(i \omega)
\end{array}\right)=\tau_{N}\left[\tau_{N} i \omega \mathbf{I}-\mathbf{Q}_{1}-\mathbf{Q}_{1}^{+} \mathbf{S}_{2}(\omega)\right]^{-1}\left\{\mathbf{C}_{1}(0)\right.
$$

$$
\left.+\sum_{n=2}^{\infty}\left[\prod_{k=2}^{n} \mathbf{Q}_{k-1}^{+} \mathbf{S}_{k}(\omega)\left(\mathbf{Q}_{k}^{-}\right)^{-1}\right] \mathbf{C}_{n}(0)\right\}
$$

where $\mathbf{I}$ is the $2 \times 2$ identity matrix, $\mathbf{Q}_{n}, \mathbf{Q}_{n}^{ \pm}$are the $2 \times 2$ matrices, given in Eqs. (3.3)-(3.5), and the matrix continued fraction $\mathbf{S}_{n}(\omega)$ is defined as 


$$
\mathbf{S}_{n}(\omega)=\left[\tau_{N} i \omega \mathbf{I}-\mathbf{Q}_{n}-\mathbf{Q}_{n}^{+} \mathbf{S}_{n+1}(\omega)\right]^{-1} \mathbf{Q}_{n}^{-} .
$$

Also

$$
\begin{aligned}
\mathbf{C}_{n}(0) & =\left(\begin{array}{c}
f_{2 n-1,1}(0) \\
f_{2 n, 1}(0)
\end{array}\right) \\
& =\left(\begin{array}{c}
\frac{n(2 n-1)}{4 n-1}\left(\left\langle P_{2 n-2}\right\rangle_{0}-\left\langle P_{2 n}\right\rangle_{0}\right) \\
\frac{n(2 n+1)}{4 n+1}\left(\left\langle P_{2 n-1}\right\rangle_{0}-\left\langle P_{2 n+1}\right\rangle_{0}\right)
\end{array}\right)
\end{aligned}
$$

In order to derive Eq. (3.8) we have used Eqs. (2.23), (2.24), and (2.29).

The initial condition vectors $\mathbf{C}_{n}(0)$ may be evaluated from the recurrence equation for the equilibrium averages of Legendre polynomials ${ }^{24}$

$$
\begin{aligned}
{[1-} & \left.\frac{2 \sigma}{(2 n-1)(2 n+3)}\right]\left\langle P_{n}\right\rangle_{0} \\
= & \frac{\xi}{2 n+1}\left[\left\langle P_{n-1}\right\rangle_{0}-\left\langle P_{n+1}\right\rangle_{0}\right] \\
& +\left[\frac{2 \sigma(n-1)}{(2 n-1)(2 n+1)}\left\langle P_{n-2}\right\rangle_{0}\right. \\
& \left.-\frac{2 \sigma(n+2)}{(2 n+1)(2 n+3)}\left\langle P_{n+2}\right\rangle_{0}\right],
\end{aligned}
$$

where the three first members of the hierarchy are given by ${ }^{24}$

$$
\left\langle P_{0}(\cos \vartheta)\right\rangle_{0}=1
$$

$$
\left\langle P_{1}(\cos \vartheta)\right\rangle_{0}=\frac{e^{\sigma} \sinh \xi}{\sigma Z}-\frac{\xi}{2 \sigma},
$$

$$
\begin{aligned}
\left\langle P_{2}(\cos \vartheta)\right\rangle_{0}= & \frac{3 e^{\sigma}}{2 \sigma Z}\left(\cosh \xi-\frac{\xi}{2 \sigma} \sinh \xi\right)+\frac{3 \xi^{2}}{8 \sigma^{2}} \\
& -\frac{3}{4 \sigma}-\frac{1}{2},
\end{aligned}
$$

and

$$
\begin{aligned}
Z= & \int_{-1}^{1} e^{\xi x+\sigma x^{2}} d x=\frac{1}{2} \sqrt{\frac{\pi}{\sigma}} e^{-\xi^{2} / 4 \sigma}\left[\operatorname{erfi}\left(\sqrt{\sigma}+\frac{\xi}{2 \sqrt{\sigma}}\right)\right. \\
& \left.+\operatorname{erfi}\left(\sqrt{\sigma}-\frac{\xi}{2 \sqrt{\sigma}}\right)\right]
\end{aligned}
$$

$[\operatorname{erfi}(x)=i \operatorname{erf}(-i x)$ is the error function of imaginary argument]. However, $\mathbf{C}_{n}(0)$ can be more efficiently evaluated by matrix continued fractions by using Risken's method. ${ }^{10}$ Namely, let us transform Eq. (3.9) to a matrix three-term recurrence relation as follows:

$$
\begin{gathered}
{ }^{0} \mathbf{Q}_{n}^{-}\left(\begin{array}{c}
\left\langle P_{2 n-3}(\cos \vartheta)\right\rangle_{0} \\
\left\langle P_{2 n-2}(\cos \vartheta)\right\rangle_{0}
\end{array}\right)+{ }^{0} \mathbf{Q}_{n}\left(\begin{array}{c}
\left\langle P_{2 n-1}(\cos \vartheta)\right\rangle_{0} \\
\left\langle P_{2 n}(\cos \vartheta)\right\rangle_{0}
\end{array}\right) \\
+{ }^{0} \mathbf{Q}_{n}^{+}\left(\begin{array}{c}
\left\langle P_{2 n+1}(\cos \vartheta)\right\rangle_{0} \\
\left\langle P_{2 n+2}(\cos \vartheta)\right\rangle_{0}
\end{array}\right)=\mathbf{0}
\end{gathered}
$$

where ${ }^{0} \mathbf{Q}_{n},{ }^{0} \mathbf{Q}_{n}^{ \pm}$are the $2 \times 2$ matrices given by

$$
\begin{gathered}
{ }^{0} \mathbf{Q}_{n}^{-}=\left(\begin{array}{cc}
\frac{4 \sigma n(n-1)(2 n-1)}{(4 n-1)(4 n-3)} & \frac{\xi n(2 n-1)}{(4 n-1)} \\
0 & \frac{2 \sigma n(2 n-1)(2 n+1)}{(4 n-1)(4 n+1)}
\end{array}\right), \\
{ }^{0} \mathbf{Q}_{n}=\left(\begin{array}{cc}
n(2 n-1)\left[\begin{array}{cc}
\frac{2 \sigma}{(4 n-3)(4 n+1)}-1
\end{array}\right] & -\frac{\xi n(2 n-1)}{(4 n-1)} \\
{ }^{0} \mathbf{Q}_{n}^{+}=\left(\begin{array}{cc}
-\frac{2 \sigma n+1)}{(4 n-1)(4 n+1)} & \\
-\frac{\xi n(2 n+1)}{(4 n+1)} & -\frac{4 \sigma n(n+1)(2 n+1)}{(4 n+1)(4 n+3)(4 n+3)}
\end{array}\right) .
\end{array}\right),
\end{gathered}
$$

In particular

$$
\left(\begin{array}{c}
\left\langle P_{1}(\cos \vartheta)\right\rangle_{0} \\
\left\langle P_{2}(\cos \vartheta)\right\rangle_{0}
\end{array}\right)=\mathbf{S}_{1}^{0}\left(\begin{array}{l}
0 \\
1
\end{array}\right)
$$

Thus, on using Eqs. (3.8) and (3.18), we are now able to

evaluate the initial conditions in terms of matrix continued

$$
\mathbf{S}_{n}^{0}=-\left[{ }^{0} \mathbf{Q}_{n}+{ }^{0} \mathbf{Q}_{n}^{+} \mathbf{S}_{n+1}^{0}\right]^{-10} \mathbf{Q}_{n}^{-}
$$

where the matrix continued fraction $\mathbf{S}_{n}^{0}$ is defined as 
fractions only, and so evaluate the complex magnetic susceptibility $\chi_{\perp}(\omega)$.

\section{RESULTS AND DISCUSSIONS}

The exact matrix continued-fraction solution [Eq. (3.6)] we have obtained is very convenient for the purpose of computation (algorithms for calculating matrix continued fractions are discussed in Ref. 10, Chap. 9). Just as for the longitudinal relaxation, ${ }^{16}$ all the matrix continued fractions and series involved converge very rapidly, thus 10-12 downward iterations in calculating the continued fractions (3.19) and 11-14 terms in the series (3.6) are enough to arrive at an accuracy not less than six significant digits in the majority cases. We remark that for $\xi=0$ the results are considerably simplified since Eq. (2.28) reduces to a scalar three-term differential-recurrence relation which has the exact solution in terms of ordinary continued fractions. ${ }^{10,12}$ The present method of solving scalar three-term recurrence relations is a particular case of the matrix one. It has been described elsewhere ${ }^{24,25}$ for similar problems and may readily be adjusted to suit the problem under consideration.

Having determined the exact solution, we may now calculate dispersion and absorption plots as well as evaluate the accuracy of various approximate solutions which have been already presented. As we have already mentioned in the Introduction, most analytical results were obtained by the effective eigenvalue method. For example, on applying this method for $\xi=0, \sigma \neq 0$, Ră̌kher and Shliomis ${ }^{22}$ derived an expression for $\chi_{\perp}(\omega)$ which in our notation is

$$
\frac{\chi_{\perp}(\omega)}{\chi_{\perp}}=\frac{\lambda_{1} \lambda_{2}+\Delta+i \omega \tau_{N} \lambda_{2}}{\left(\lambda_{2}+i \omega \tau_{N}\right)\left(\lambda_{1}+i \omega \tau_{N}\right)+\Delta},
$$

where $\Delta=\sigma \alpha^{-2}\left(\lambda_{1}-1\right)$. Here $\lambda_{1}, \lambda_{2}$ are the effective eigenvalues which can be expressed in terms of equilibrium values of the Legendre polynomial of order $2,\left\langle P_{2}\right\rangle_{0}$, viz.

$$
\lambda_{1}=\frac{1+\frac{1}{2}\left\langle P_{2}\right\rangle_{0}}{1-\left\langle P_{2}\right\rangle_{0}}, \quad \lambda_{2}=\frac{2 \sigma+\left\langle P_{2}\right\rangle_{0}(\sigma-6)}{3\left\langle P_{2}\right\rangle_{0}}
$$

with

$$
\left\langle P_{2}\right\rangle_{0}=\frac{3 e^{\sigma}}{2 \sqrt{\pi \sigma} \operatorname{erfi}(\sqrt{\sigma})}-\frac{3}{4 \sigma}-\frac{1}{2} .
$$

For high potential barriers $(\sigma \gg 1)$ when

$$
\lambda_{1} \sim \sigma, \quad \lambda_{2} \sim \sigma, \quad \Delta \sim \sigma^{2} \alpha^{-2},
$$

Eq. (4.1) reduces ${ }^{22}$ to the ferromagnetic resonance equation of Landau and Lifshitz ${ }^{5}$

$$
\frac{\chi_{\perp}(\omega)}{\chi_{\perp}}=\frac{\left(1+\alpha^{2}\right) \omega_{0}^{2}+i \alpha \omega \omega_{0}}{\left(1+\alpha^{2}\right) \omega_{0}^{2}-\omega^{2}+2 i \alpha \omega \omega_{0}},
$$

where

$$
\omega_{0}=\sigma\left(\alpha \tau_{N}\right)^{-1} \approx \gamma H_{a n},
$$

and $H_{a n}=2 K / M_{s}$ is the strength of the anisotropy field.

Furthermore, on using the effective eigenvalue method in the opposite limit $\sigma=0, \xi \neq 0$, Garanin, Ischenko, and
Panina ${ }^{15}$ obtained an equation for the circular magnetic susceptibility, which can be rearranged to the following expression for the complex transverse susceptibility:

$$
\frac{\chi_{\perp}(\omega)}{\chi_{\perp}}=\frac{|\lambda|^{2}+i \omega \tau_{N} \lambda^{\prime}}{|\lambda|^{2}-\omega^{2} \tau_{N}^{2}+2 i \omega \tau_{N} \lambda^{\prime}},
$$

where $\lambda$ is the effective eigenvalue (now complex) given by

$$
\lambda=\lambda^{\prime}+i \lambda^{\prime \prime}=\frac{\xi^{2}+1-\xi \operatorname{coth} \xi}{2(\xi \operatorname{coth} \xi-1)}+\frac{i \xi}{2 \alpha} .
$$

For high-field parameters ( $\xi \gg 1)$ Eq. (4.6) also reduces to the Landau-Lifshitz equation (4.4) with $\omega_{0}=\xi\left(2 \alpha \tau_{N}\right)^{-1}$. Equation (4.7) was derived for $\xi \neq 0, \sigma=0$ only. However, on noting that $\lambda$ is the effective eigenvalue for the equilibrium autocorrelation function $f_{1,1}(t)$ as well, Eqs. (4.6) and (4.7) can readily be generalized for $\sigma \approx 0$ and/or $\sigma \ll \xi$ as follows. According to Eq. (2.25), the first equation of the infinite hierarchy is

$$
\begin{aligned}
\tau_{N} \frac{d}{d t} f_{1,1}(t)+\left[1+\frac{i \xi}{2 \alpha}+\frac{\sigma}{5}\right] f_{1,1}(t)+\left(\frac{i \sigma}{3 \alpha}+\frac{\xi}{6}\right) f_{2,1}(t) \\
+\frac{2 \sigma}{15} f_{3,1}(t)=0
\end{aligned}
$$

The effective eigenvalue approach implies that the solution of Eq. (4.8) is approximated by a single exponential, namely, $f_{1,1}(t)=f_{1,1}(0) e^{-\lambda t / \tau_{N}}$ with the effective eigenvalue $\lambda$ given by

$$
\begin{aligned}
\lambda=-\frac{\dot{f}_{1,1}(0)}{f_{1,1}(0)}= & 1+\frac{i \xi}{2 \alpha}+\frac{\sigma}{5}+\left(\frac{i \sigma}{3 \alpha}+\frac{\xi}{6}\right) \frac{f_{2,1}(0)}{f_{1,1}(0)} \\
& +\frac{2 \sigma}{15} \frac{f_{3,1}(0)}{f_{1,1}(0)} .
\end{aligned}
$$

Equation (4.9) can be simplified after some algebra to yield

$\lambda=\lambda^{\prime}+i \lambda^{\prime \prime}=\frac{2+\left\langle P_{2}\right\rangle_{0}}{2\left(1-\left\langle P_{2}\right\rangle_{0}\right)}+i \frac{3\left\langle P_{1}\right\rangle_{0}}{2 \alpha\left(1-\left\langle P_{2}\right\rangle_{0}\right)}$,

where $\left\langle P_{1}\right\rangle_{0}$ and $\left\langle P_{2}\right\rangle_{0}$ are given by Eqs. (3.11) and (3.12), respectively. For $\sigma=0$, Eq. (4.10) reduces to Eq. (4.8) since in this case

$$
\left\langle P_{1}\right\rangle_{0}=\operatorname{coth} \xi-\xi^{-1}, \quad\left\langle P_{2}\right\rangle_{0}=1-3 \xi^{-1}\left(\operatorname{coth} \xi-\xi^{-1}\right) .
$$

The generalization of the Răkher and Shliomis results ${ }^{22}$ for $\xi \neq 0, \xi \ll \sigma$ is given in Appendix B. Moreover, for arbitrary $\xi$ and $\sigma$ and large damping (when one may ignore precessional motion) the transverse spectrum may effectively be described by the Debye relaxational equation ${ }^{19}$

$$
\frac{\chi_{\perp}(\omega)}{\chi_{\perp}}=\frac{1}{1+i \omega \tau_{N} / \lambda^{\prime}},
$$




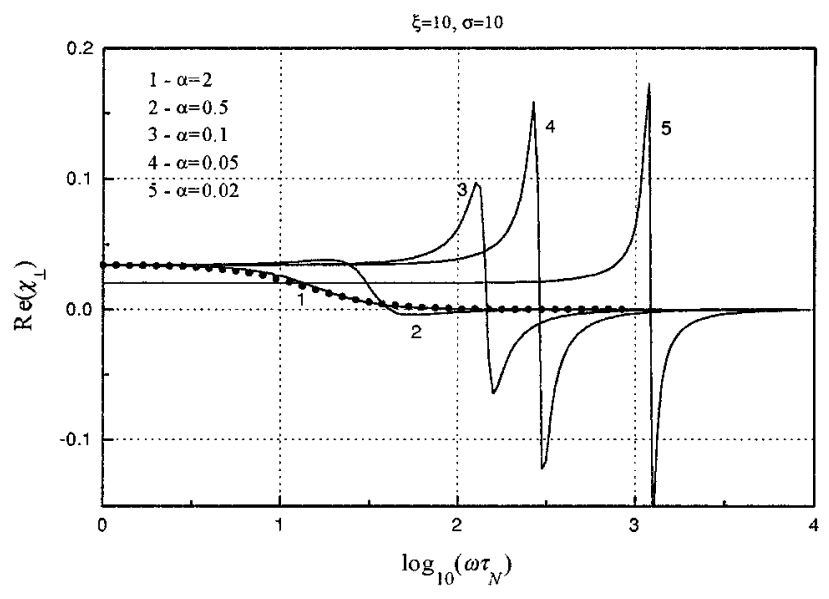

FIG. 1. The real part of $\chi_{\perp}(\omega)$ (solid lines) vs $\log _{10}\left(\omega \tau_{N}\right)$ for $\sigma=10$ and $\xi=10$ and various values of $\alpha$. Filled circles are the overdamped effective eigenvalue solution from Eqs. (4.12) and (4.13).

where $\lambda^{\prime}$ is the effective eigenvalue which can also be expressed in terms of $\left\langle P_{2}\right\rangle_{0}$ from Eq. (3.12), viz. [cf. Eqs. (4.2) and (4.10)]

$$
\lambda^{\prime}=\frac{1+1 / 2\left\langle P_{2}\right\rangle_{0}}{1-\left\langle P_{2}\right\rangle_{0}}
$$

It should be noted that Eqs. (4.10) and (4.13) are valid for any uniaxial potential. ${ }^{19}$

Typical spectra of the real and imaginary parts of $\chi_{\perp}(\omega)$ are shown in Figs. 1-6 (the calculations were carried out for $\left.\nu^{2} M_{s}^{2} N_{0} / k T=1\right)$. The relaxational behavior of the spectra $\chi_{\perp}(\omega)$ is obtained for a small anisotropy and field parameters $(\xi, \sigma \approx 0)$ or large damping. s expected at small damping the spectra have a pronounced resonant character and strongly depend on the damping parameter $\alpha$ (Figs. 1 and 2). However, the value of $\alpha$ remains unknown. Methods of experimental and theoretical estimations of $\alpha$ were discussed, e.g., in Refs. 21, 22, and 26, but no experimental data

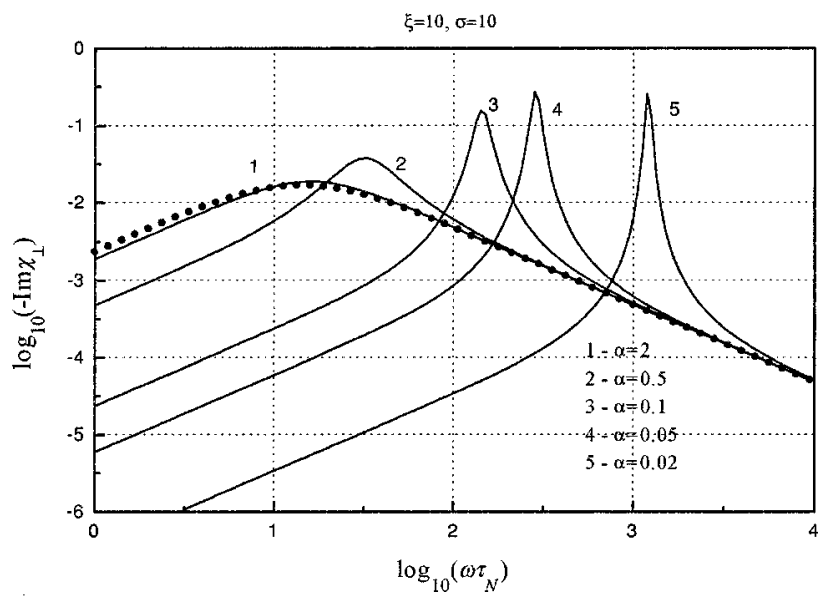

FIG. 2. $\log _{10}\left(-\operatorname{Im}\left\{\chi_{\perp}(\omega\}\right)\right.$ (solid lines) vs $\log _{10}\left(\omega \tau_{N}\right)$ for $\sigma=10$ and $\xi=10$ and various values of $\alpha$. Filled circles are the overdamped effective eigenvalue solution from Eqs. (4.12) and (4.13).

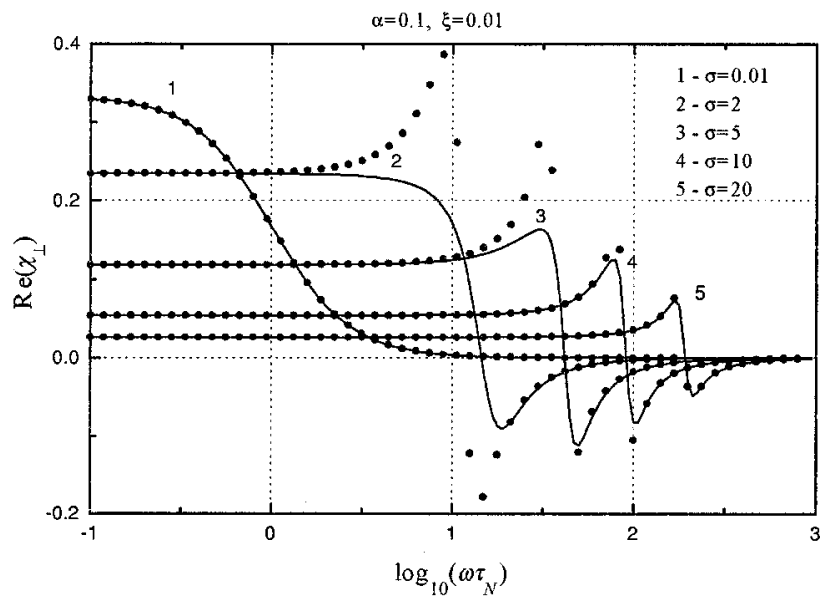

FIG. 3. Comparison of the exact (solid lines) and effective eigenvalue [filled circles, Eqs. (2.31) and (B7)] solutions for the real part of $\chi_{\perp}(\tau)$ vs $\log _{10}\left(\omega \tau_{N}\right)$ for $\alpha=0.1$ and $\xi=0.01$ and various values of $\sigma$.

seem to be available. Some theoretical estimations of $\alpha$ yield values of order of 0.01-0.1.22 The comparison of the effective eigenvalue solutions ${ }^{15,19,21}$ with the exact results allows us to estimate the accuracy of the former. In Figs. 1 and 2 the results of the exact calculations are compared with the overdamped solution Eq. (4.12). ${ }^{19}$ In Figs. 3 and 4 the exact calculations are compared with the solution of Raikher and Shliomis. ${ }^{22}$ It is obviously by inspection of these figures that both solutions are in agreement for $\sigma \approx 0$ and for $\sigma \gg 1$ only. However, in the most interesting range of the barrier height parameter $\sigma \sim 1-5$, the effective eigenvalue approach fails to describe the transverse response. The explanation appears to be as follows: at small to moderate barrier heights there is essentially a spread of the precession frequencies of the magnetization in the anisotropy field. To a certain extent this effect is analogous to inhomogeneous broadening and considerably exceeds the true damping. ${ }^{15}$ Therefore, it is practically impossible to describe asymmetric absorption and dis-

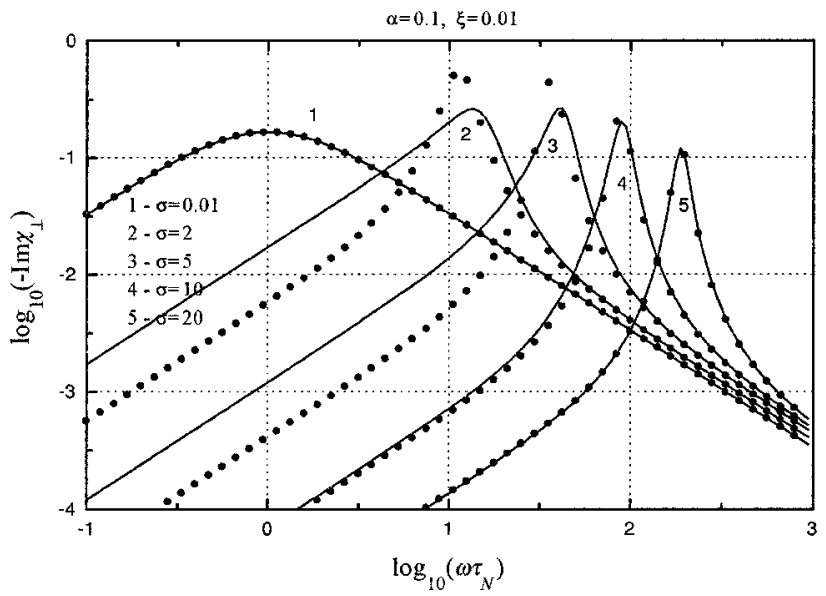

FIG. 4. Comparison of the exact (solid lines) and effective eigenvalue [filled circles, Eqs. (2.31) and (B7)] solutions for $\log _{10}$ $\left(-\operatorname{Im}\left\{\chi_{\perp}(\omega\}\right)\right.$ vs $\log _{10}\left(\omega \tau_{N}\right)$ for $\alpha=0.1$ and $\xi=0.01$ and various values of $\sigma$. 


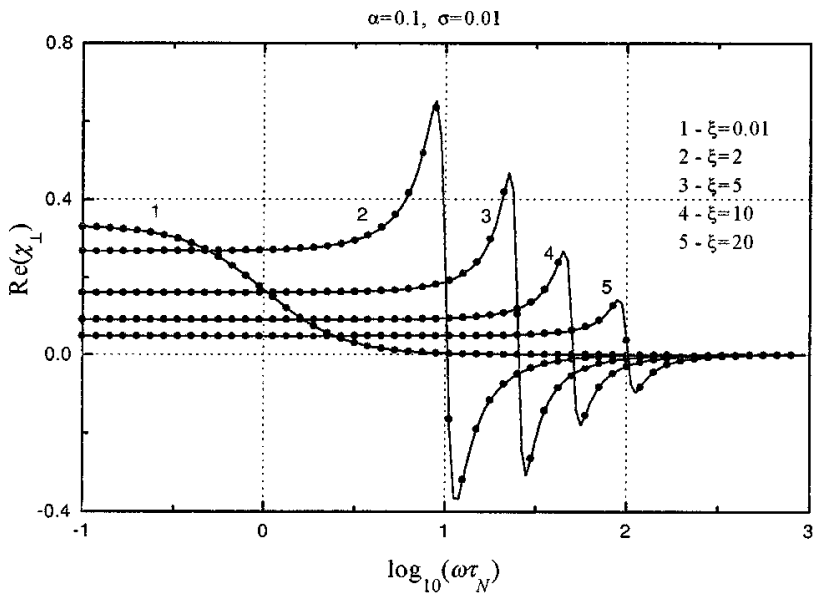

FIG. 5. Comparison of the exact (solid lines) and effective eigenvalue [filled circles, Eqs. (4.6) and (4.10)] solutions for the real part of $\chi_{\perp}(\omega)$ vs $\log _{10}\left(\omega \tau_{N}\right)$ for $\alpha=0.1$ and $\sigma=0.01$ and various values of $\xi$.

persion curves by the usual single resonance spectrum which is predicted by the effective eigenvalue approach. However, in the high barrier limit ( $\sigma \gg 1)$ when the anisotropy potential may be approximated by a harmonic potential the system may be effectively described by a single resonance with the characteristic frequency $\omega_{0}$ given by Eq. (4.5). On the other hand, for $\sigma \approx 0$ and/or $\sigma \ll \xi$ the effective eigenvalue approach provides (just as for the longitudinal relaxation ${ }^{16}$ ) perfect correspondence with the exact solution for all values of the field parameter $\xi$ (see Figs. 5 and 6) since we now have the natural resonance with the angular frequency $\omega_{0}$ $=\xi\left(2 \alpha \tau_{N}\right)^{-1}$. Thus, when the external magnetic field considerably exceeds the anisotropy field or the anisotropy field is close to zero, the effective eigenvalue method accurately describes the transverse relaxation. However, when the influence of a constant magnetic field is negligible, the effective eigenvalue method requires careful investigation of the range of its applicability before proceeding. Otherwise, it is pos-

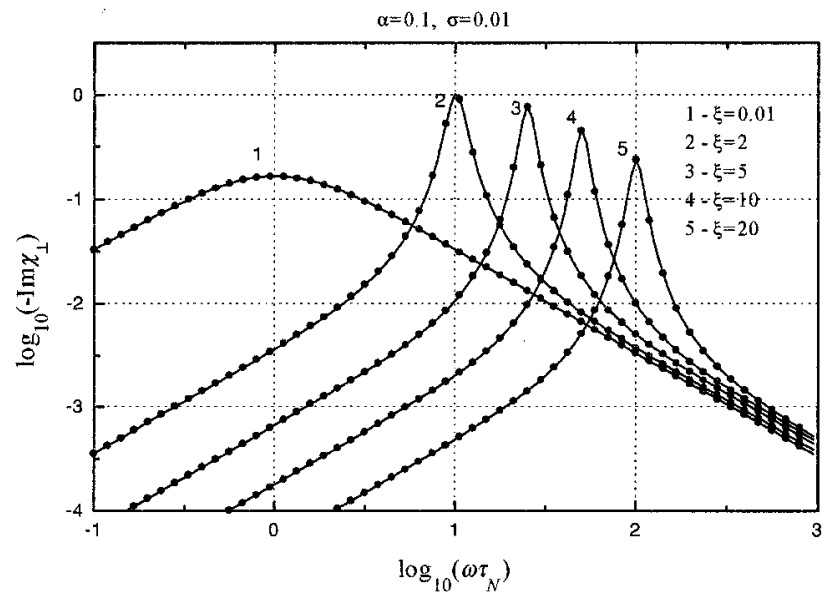

FIG. 6. Comparison of the exact (solid lines) and effective eigenvalue [filled circles, Eqs. (4.6) and (4.10)] solutions for $\log _{10}$ $\left(-\operatorname{Im}\left\{\chi_{\perp}(\omega\}\right)\right.$ vs $\log _{10}\left(\omega \tau_{N}\right)$ for $\alpha=0.1$ and $\sigma=0.01$ and various values of $\xi$. sible to arrive at erroneous results as clearly demonstrated in Figs. 3 and 4. We remark that for the longitudinal relaxation the effective eigenvalue approach has a restricted range of validity and is applicable for low barrier heights $(\sigma \leqslant 1)$ only.

Thus, the transverse response of an ensemble of noninteracting single domain particles can be evaluated from the $e x$ act equation (3.6). Furthermore, we demonstrated that the effective eigenvalue approach which yields simple analytical expressions [Eqs. (4.1), (4.6), (4.10), (4.12), (B6)], describes the main features of the transverse complex susceptibility with the exception of the range of intermediate barriers $\sigma$ $\sim 1-5$ and $\xi \leqslant 1$, where there exists essentially a spread of the precession frequencies of the magnetization in the anisotropy potential field. In order to derive these results we have assumed that all particles are identical. This assumption is practically never fulfilled in an experiment. In order to take into account the polydispersity of the particles one must also average the susceptibility over appropriate distribution functions (e.g., over that of particle volumes). However, as demonstrated by Shliomis and Stepanov ${ }^{31}$ such averaging does not substantially alter the transverse susceptibility in contrast to the longitudinal one where the averaging may considerably change the spectrum. In order to simplify the theory we have also assumed throughout that the constant magnetic field $H_{0}$ is applied along the easy axis of the magnetization. When $H_{0}$ is at an arbitrary angle to that axis the theory becomes very much more complicated. ${ }^{17}$ The results of such calculations of the transverse response are described in Ref. 32. Equations (4.6) and (4.7) were first derived in Ref. 33.

\section{ACKNOWLEDGMENTS}

The partial support of this work by the Russian Foundation for Basic Research (Grant No. 96-02-16762- $a$ ) and the Forbairt Research Collaboration Fund is gratefully acknowledged.

\section{APPENDIX A}

Noting that the rule for changing of variables in Stratonovich differential equations is the same as in ordinary analysis, ${ }^{27}$ the equation of motion for an arbitrary differentiable function $f(\{\xi\})$ may be obtained by cross multiplying $i$ th Eq. (2.10) by $\partial f(\{\xi(t)\}) / \partial \xi_{i}$, respectively, and then summing them. Thus, we obtain a stochastic equation for $f(\{\xi(t)\})$ :

$$
\begin{aligned}
\frac{d}{d t} f(\{\xi(t)\})= & h_{i}(\{\xi(t)\}, t) \frac{\partial}{\partial \xi_{i}} f(\{\xi(t)\}) \\
& +g_{i j}(\{\xi(t)\}, t) \frac{\partial}{\partial \xi_{i}} f(\{\xi(t)\}) \Gamma_{j}(t) .
\end{aligned}
$$

From a mathematical point of view the stochastic differential equation (A1) [just as Eq. (2.10)] with the $\delta$-correlated Langevin forces $\Gamma_{j}(t)$ is not completely defined. ${ }^{10,27}$ The 
most satisfactory interpretation of Eqs. (2.10) and (A1) is as the stochastic integral equations ${ }^{10,12}$

$$
\begin{aligned}
\xi_{i}(t+\tau)= & x_{i}+\int_{t}^{t+\tau} h_{i}\left(\left\{\xi\left(t^{\prime}\right)\right\}, t^{\prime}\right) d t^{\prime} \\
& +\int_{t}^{t+\tau} g_{i j}\left(\left\{\xi\left(t^{\prime}\right)\right\}, t^{\prime}\right) \Gamma_{j}\left(t^{\prime}\right) d t^{\prime},
\end{aligned}
$$

$$
\begin{aligned}
f(\{\xi(t+\tau)\})= & f(\{\mathbf{x}\})+\int_{t}^{t+\tau} h_{i}\left(\left\{\xi\left(t^{\prime}\right)\right\}, t^{\prime}\right) \frac{\partial f\left(\left\{\xi\left(t^{\prime}\right)\right\}\right)}{\partial \xi_{i}} d t^{\prime} \\
& +\int_{t}^{t+\tau} g_{i j}\left(\left\{\xi\left(t^{\prime}\right)\right\}, t^{\prime}\right) \frac{\partial f\left(\left\{\xi\left(t^{\prime}\right)\right\}\right)}{\partial \xi_{i}} \Gamma_{j}\left(t^{\prime}\right) d t^{\prime} .
\end{aligned}
$$

On supposing that the integrands in Eqs. (A2) and (A3) can be expanded in Taylor series, we obtain

$$
\begin{aligned}
\xi_{i}(t+\tau)= & x_{i}+\int_{t}^{t+\tau} h_{i}\left(\{\mathbf{x}\}, t^{\prime}\right) d t^{\prime}+\int_{t}^{t+\tau}\left[\xi_{k}\left(t^{\prime}\right)-x_{k}\right] \frac{\partial}{\partial x_{k}} h_{i}\left(\{\mathbf{x}\}, t^{\prime}\right) d t^{\prime}+\int_{t}^{t+\tau} g_{i j}\left(\{\mathbf{x}\}, t^{\prime}\right) \Gamma_{j}\left(t^{\prime}\right) d t^{\prime}+\int_{t}^{t+\tau}\left[\xi_{k}\left(t^{\prime}\right)-x_{k}\right] \\
& \times \frac{\partial}{\partial x_{k}} g_{i j}\left(\{\mathbf{x}\}, t^{\prime}\right) \Gamma_{j}\left(t^{\prime}\right) d t^{\prime}+\cdots, \\
f(\{\xi(t+\tau)\})= & f(\{\mathbf{x}\})+\int_{t}^{t+\tau} h_{i}\left(\{\mathbf{x}\}, t^{\prime}\right) \frac{\partial f(\{\mathbf{x}\})}{\partial x_{i}} d t^{\prime}+\int_{t}^{t+\tau}\left[\xi_{k}\left(t^{\prime}\right)-x_{k}\right] \frac{\partial}{\partial x_{k}}\left[h_{i}\left(\{\mathbf{x}\}, t^{\prime}\right) \frac{\partial f(\{\mathbf{x}\})}{\partial x_{i}}\right] d t^{\prime} \\
& +\int_{t}^{t+\tau} g_{i j}\left(\{\mathbf{x}\}, t^{\prime}\right) \frac{\partial f(\{\mathbf{x}\})}{\partial x_{i}} \Gamma_{j}\left(t^{\prime}\right) d t^{\prime}+\int_{t}^{t+\tau}\left[\xi_{k}\left(t^{\prime}\right)-x_{k}\right] \frac{\partial}{\partial x_{k}}\left[g_{i j}\left(\{\mathbf{x}\}, t^{\prime}\right) \frac{\partial f(\{\mathbf{x}\})}{\partial x_{i}}\right] \Gamma_{j}\left(t^{\prime}\right) d t^{\prime}+\cdots .
\end{aligned}
$$

On substituting $\xi_{k}\left(t^{\prime}\right)-x_{k}$ from Eq. (A4) into Eq. (A5) we iterate

$$
\begin{aligned}
f(\{\xi(t+\tau)\})= & f(\{\mathbf{x}\})+\int_{t}^{t+\tau} h_{i}\left(\{\mathbf{x}\}, t^{\prime}\right) \frac{\partial f(\{\mathbf{x}\})}{\partial x_{i}} d t^{\prime}+\int_{t}^{t+\tau} \frac{\partial}{\partial x_{k}}\left[h_{i}\left(\{\mathbf{x}\}, t^{\prime}\right) \frac{\partial f(\{\mathbf{x}\})}{\partial x_{i}}\right] \int_{t}^{t^{\prime}} h_{k}\left(\{\mathbf{x}\}, t^{\prime \prime}\right) d t^{\prime \prime} d t^{\prime} \\
& +\int_{t}^{t+\tau} \frac{\partial}{\partial x_{k}}\left[h_{i}\left(\{\mathbf{x}\}, t^{\prime}\right) \frac{\partial f(\{\mathbf{x}\})}{\partial x_{i}}\right] \int_{t}^{t^{\prime}} g_{k n}\left(\{\mathbf{x}\}, t^{\prime \prime}\right) \Gamma_{n}\left(t^{\prime \prime}\right) d t^{\prime \prime} d t^{\prime}+\int_{t}^{t+\tau} g_{i j}\left(\{\mathbf{x}\}, t^{\prime}\right) \frac{\partial f(\{\mathbf{x}\})}{\partial x_{i}} \Gamma_{j}\left(t^{\prime}\right) d t^{\prime} \\
& +\int_{t}^{t+\tau} \frac{\partial}{\partial x_{k}}\left[g_{i j}\left(\{\mathbf{x}\}, t^{\prime}\right) \frac{\partial f(\{\mathbf{x}\})}{\partial x_{i}}\right] \Gamma_{j}(t)^{\prime} \int_{t}^{t^{\prime}} h_{k}\left(\{\mathbf{x}\}, t^{\prime \prime}\right) d t^{\prime \prime} d t^{\prime} \\
& +\int_{t}^{t+\tau} \frac{\partial}{\partial x_{k}}\left[g_{i j}\left(\{\mathbf{x}\}, t^{\prime}\right) \frac{\partial f(\{\mathbf{x}\})}{\partial x_{i}}\right] \Gamma_{j}\left(t^{\prime}\right) \int_{t}^{t^{\prime}} g_{k n}\left(\{\mathbf{x}\}, t^{\prime \prime}\right) \Gamma_{n}\left(t^{\prime \prime}\right) d t^{\prime \prime} d t^{\prime}+\cdots
\end{aligned}
$$

Then averaging Eq. (A6) with account of the properties (2.11) and retaining only the terms of the order of $\tau$, we have

$$
\begin{aligned}
\overline{f(\{\xi(t+\tau)\})}= & f(\{\mathbf{x}\})+\int_{t}^{t+\tau} h_{i}\left(\{\mathbf{x}\}, t^{\prime}\right) \frac{\partial}{\partial x_{i}} f(\{\mathbf{x}\}) d t^{\prime}+2 D \delta_{j n} \int_{t}^{t+\tau} \frac{\partial}{\partial x_{k}}\left[g_{i j}\left(\{\mathbf{x}\}, t^{\prime}\right) \frac{\partial}{\partial x_{i}} f(\{\mathbf{x}\})\right] \int_{t}^{t^{\prime}} g_{k n}\left(\{\mathbf{x}\}, t^{\prime \prime}\right) \\
& \times \delta\left(t^{\prime}-t^{\prime \prime}\right) d t^{\prime \prime} d t^{\prime}+o(\tau) .
\end{aligned}
$$

After obvious transformations in Eq. (A7), we obtain

$$
\frac{\overline{f(\{\xi(t+\tau)\})}-f(\{\mathbf{x}\})}{\tau}=h_{i}\left(\{\mathbf{x}\}, t+\tau \Theta_{i i i}^{(1)}\right) \frac{\partial}{\partial x_{i}} f(\{\mathbf{x}\})+D g_{k j}\left(\{\mathbf{x}\}, t+\tau \Theta_{i j k}^{(2)}\right) \frac{\partial}{\partial x_{k}}\left[g_{i j}\left(\{x\}, t+\tau \Theta \Theta_{i j k}^{(2)}\right) \frac{\partial}{\partial x_{i}} f(\{\mathbf{x}\})\right]+o(1),
$$

where $\Theta_{i j k}^{(n)}$ are constants $\left(0 \leqslant \Theta_{i j k}^{(n)} \leqslant 1\right)$. Here we have also used the property of the $\delta$ function

$$
y(b)=2 \int_{a}^{b} \delta(b-x) y(x) d x
$$

Taking the limit $\tau \rightarrow 0$ in Eq. (A8), we have Eq. (2.13). 


\section{APPENDIX B}

The application of the effective eigenvalue approach at $\xi \neq 0, \xi \ll \sigma$ requires two effective eigenvalues for the first two equations of the infinite hierarchy Eq. (2.25), since the resonance arises here due to the coupling between $f_{1,1}(t)$ and $f_{2,1}(t)$ (this behavior is termed "entanglement" of the dipole and quadrupole branches of the response by Răkher and Shliomis). ${ }^{22}$ Thus we obtain the set of coupled equations, viz.

$$
\begin{aligned}
\tau_{N} \frac{d}{d t} f_{1,1}(t)+\lambda_{1}^{\mathrm{eff}} f_{1,1}(t)+\left(\frac{i \sigma}{3 \alpha}+\frac{\xi}{6}\right) f_{2,1}(t)=0, \quad(\mathrm{~B} 1) \\
\tau_{N} \frac{d}{d t} f_{2,1}(t)+\lambda_{2}^{\mathrm{eff}} f_{2,1}(t)-3\left[\left(1-\lambda_{1}^{\mathrm{eff}}\right)\left(\frac{\xi}{\sigma}+\frac{i}{\alpha}\right)+\frac{\xi}{2}\right] f_{1,1}(t) \\
=0,
\end{aligned}
$$

where the effective eigenvalues are given by

$$
\begin{aligned}
\lambda_{1}^{\mathrm{eff}} & =1+\frac{i \xi}{2 \alpha}+\frac{\sigma}{5}+\frac{2 \sigma}{15} \frac{f_{3,1}(0)}{f_{1,1}(0)} \\
& =\frac{i \xi}{2 \alpha}+\frac{\xi^{2}}{4 \sigma}-\frac{1}{2}+\frac{3}{4 \sigma}\left(\frac{2 \sigma-\xi\left\langle P_{1}\right\rangle_{0}}{1-\left\langle P_{2}\right\rangle_{0}}\right), \\
\lambda_{2}^{\mathrm{eff}}= & \frac{i \xi}{2 \alpha}+3-\frac{\sigma}{7}+\frac{12 \sigma}{35} \frac{f_{4,1}(0)}{f_{2,1}(0)}=\frac{i \xi}{2 \alpha}-1-\sigma+\frac{\xi^{2}}{2 \sigma} \\
& +3\left[\frac{2 \sigma\left\langle P_{1}\right\rangle_{0}-\xi\left\langle P_{2}\right\rangle_{0}}{\xi\left(\left\langle P_{2}\right\rangle_{0}-1\right)+3\left\langle P_{1}\right\rangle_{0}}\right] .
\end{aligned}
$$

Here $\left\langle P_{1}\right\rangle_{0}$ and $\left\langle P_{2}\right\rangle_{0}$ are given by Eqs. (3.11) and (3.12), respectively. At the derivation of $\mathrm{Eq}$. (B2) we have taken into account that

$$
f_{3,1}(t)=\left(\lambda_{1}^{\mathrm{eff}}-1-\frac{i \xi}{2 \alpha}-\frac{\sigma}{5}\right) \frac{15}{2 \sigma} f_{1,1}(t)
$$

[in accordance with Eq. (B3) the equality (B5) is assumed to be valid at any time $t]$. We note that in the limit $\xi, \sigma \rightarrow 0$ the effective eigenvalues have the following behavior:

$$
\begin{aligned}
& \lambda_{1}^{\mathrm{eff}} \approx \frac{i \xi}{2 \alpha}+1+\frac{\sigma}{5}+\frac{8 \sigma^{2}}{175}+\frac{4 \sigma \xi^{2}}{175}+\cdots, \\
& \lambda_{2}^{\mathrm{eff}} \approx \frac{i \xi}{2 \alpha}+3-\frac{\sigma}{7}+\frac{16 \sigma^{2}}{147}+\frac{8 \sigma \xi^{2}}{441}+\cdots .
\end{aligned}
$$

On applying the one-sided Fourier transform to Eqs. (B1) and (B2), we can solve the set of linear equations so obtained for $\widetilde{f}_{1,1}(i \omega)$ yielding

$$
\frac{\widetilde{f}_{1,1}(i \omega)}{f_{1,1}(0)}=\frac{\left(\lambda_{1}^{\mathrm{eff}}+i \omega \tau_{N}\right)-\delta}{\left(\lambda_{1}^{\mathrm{eff}}+i \omega \tau_{N}\right)\left(\lambda_{2}^{\mathrm{eff}}+i \omega \tau_{N}\right)+\Delta},
$$

where

$$
\begin{gathered}
\Delta=\left(\frac{\xi}{2}+\frac{i \sigma}{\alpha}\right)\left[\left(1-\lambda_{1}^{\mathrm{eff}}\right)\left(\frac{\xi}{\sigma}+\frac{i}{\alpha}\right)+\frac{\xi}{2}\right], \\
\delta=\left(\frac{\xi}{6}+\frac{i \sigma}{3 \alpha}\right) \frac{f_{2,1}(0)}{f_{1,1}(0)}=\left(\frac{\xi}{4 \sigma}+\frac{i}{2 \alpha}\right)\left(\frac{3\left\langle P_{1}\right\rangle_{0}}{1-\left\langle P_{2}\right\rangle_{0}}-\xi\right) .
\end{gathered}
$$

On substituting Eq. (B6) into Eq. (2.31), we can evaluate $\chi_{\perp}(\omega)$, which at $\xi=0$ reduces to Eq. (4.1).
*Author to whom correspondence should be addressed.

${ }^{1}$ L. Néel, Ann. Geophys. (France) 5, 99 (1949).

${ }^{2}$ W. F. Brown, Jr., IEEE Trans. Magn. 15, 1196 (1979).

${ }^{3}$ H. B. Braun and H. N. Bertram, J. Appl. Phys. 75, 4609 (1994).

${ }^{4}$ C. P. Bean and J. D. Livingston, J. Appl. Phys. Suppl. 30, 1205 (1959).

${ }^{5}$ L. D. Landau and E. M. Lifshitz, Phys. Z. Sowjetunion 8, 153 (1935).

${ }^{6}$ T. L. Gilbert, Phys. Rev. 100, 1243 (1956) (Abstract only).

${ }^{7}$ W. F. Brown, Jr., Phys. Rev. 130, 1677 (1963); J. Appl. Phys. Suppl. 30, 130S (1959).

${ }^{8}$ M. C. Wang and G. E. Uhlenbeck, Rev. Mod. Phys. 17, 323 (1945).

${ }^{9}$ R. L. Stratonovich, Conditional Markov Processes and Their Application to the Theory of Optimal Control (Elsevier, New York, 1968).

${ }^{10}$ H. Risken, The Fokker-Planck Equation, 2nd ed. (Springer, Berlin, 1989).

${ }^{11}$ Yu. P. Kalmykov, J. Mol. Liq. 69, 117 (1996).

${ }^{12}$ W. T. Coffey, Yu. P. Kalmykov, and J. T. Waldron, The Langevin Equation (Singapore, World Scientific, 1996).

${ }^{13}$ A. Aharoni, Phys. Rev. 177, 763 (1969).

${ }^{14}$ E. C. Stoner and E. P. Wohlfahrt, Philos. Trans. R. Soc. London, Ser. A 240, 599 (1948).
${ }^{15}$ D. A. Garanin, V. V. Ischenko, and L. V. Panina, Teor. Mat. Fiz. 82, 242 (1990). [ Theor. Math. Phys. 82, 169 (1990)].

${ }^{16}$ W. T. Coffey, D. S. F. Crothers, Yu. P. Kalmykov, and J. T. Waldron, Phys. Rev. B 51, 15947 (1995).

${ }^{17}$ W. T. Coffey, D. S. F. Crothers, J. L. Dormann, L. J. Geoghegan, Yu. P. Kalmykov, J. T. Waldron, and A. W. Wickstead, Phys. Rev. B 52, 15951 (1995).

${ }^{18}$ D. A. Garanin, Phys. Rev. E 54, 3250 (1996).

${ }^{19}$ W. T. Coffey, P. J. Cregg, and Yu. P. Kalmykov, Advances in Chemical Physics, edited by I. Prigogine and S. A. Rice (Wiley, New York, 1993), Vol. 83, pp. 263-464.

${ }^{20}$ W. T. Coffey, Yu. P. Kalmykov, and E. S. Massawe, Advances in Chemical Physics, edited by I. Prigogine and S. A. Rice (Wiley, New York, 1993), Vol. 85, Part 2, p. 667.

${ }^{21}$ Yu. L. Raǐkher and M. I. Shliomis, Advances in Chemical Physics, edited by W. T. Coffey, I. Prigogine, and S. A. Rice (Wiley, New York, 1994), Vol. 87, p. 595.

${ }^{22}$ Yu. L. Raŭkher and M. I. Shliomis, Zh. Eksp. Teor. Fiz. 67, 1060 (1974) [ Sov. Phys. JETP 40, 526 (1974]).

${ }^{23}$ Yu. L. Raĭkher and V. I. Stepanov, Zh. Eksp. Teor. Fiz. 102, 1409 (1992) [ Sov. Phys. JETP 75, 764 (1995)].

${ }^{24}$ W. T. Coffey, J. L. Déjardin, Yu. P. Kalmykov, and S. V. Titov, Phys. Rev. E 54, 6462 (1996).

${ }^{25}$ J. L. Déjardin, P. M. Déjardin, and Yu. P. Kalmykov, J. Chem. Phys. 106, 5824 (1997). 
${ }^{26}$ I. Klik and L. Gunther, J. Stat. Phys. 60, 473 (1990).

${ }^{27}$ C. W. Gardiner, Handbook of Stochastic Methods (Springer, Berlin, 1985).

${ }^{28}$ Handbook of Mathematical Functions, edited by M. Abramowitz and I. Stegun (Dover, New York, 1965).

${ }^{29}$ A. R. Edmonds, Angular Momentum in Quantum Mechanics (Princeton University Press, Princeton, 1957).

${ }^{30}$ W. T. Coffey, Yu. P. Kalmykov, and J. T. Waldron, Physica A
208, 462 (1994).

${ }^{31}$ M. I. Shliomis and V. I. Stepanov, Advances in Chemical Physics (Ref. 21), Vol. 87, p. 1.

${ }^{32}$ W. T. Coffey, D. S. F. Crothers, J. L. Dormann, L. J. Geoghegan, J. T. Waldron, and E. Kennedy (unpublished).

${ }^{33}$ R. S. Gekht, V. A. Ignatchenko, Yu. L. Raĭkher, and M. I. Shliomis, Zh. Eksp. Teor. Fiz. 70, 1290 (1976) [Sov. Phys. JETP 43, 677 (1976)]. 